Research Article

\title{
Differential Measurement for Cavity Ring-Down Spectroscopy with Dynamic Allan Variance
}

\author{
Zeqiang Mo, ${ }^{1,2,3}$ Jin Yu $\mathbb{D}^{1},{ }^{1,2}$ Jinduo Wang, ${ }^{1,2}$ Jianguo He, ${ }^{1,2,3}$ Shoujun Dai, ${ }^{1,2}$ and Yang Liu ${ }^{1,3}$ \\ ${ }^{1}$ Aerospace Information Research Institute, Chinese Academy of Sciences, Beijing 100094, China \\ ${ }^{2}$ School of Optoelectronics, University of Chinese Academy of Sciences, Beijing 100049, China \\ ${ }^{3}$ Key Laboratory of Computational Optical Imaging Technology, Chinese Academy of Sciences, Beijing 100094, China
}

Correspondence should be addressed to Jin Yu; jinyu@aoe.ac.cn

Received 6 February 2020; Accepted 7 March 2020; Published 14 April 2020

Academic Editor: Young Jong Lee

Copyright ( $\odot 2020$ Zeqiang Mo et al. This is an open access article distributed under the Creative Commons Attribution License, which permits unrestricted use, distribution, and reproduction in any medium, provided the original work is properly cited.

The method of dynamic Allan variance (DAVAR) is used to analyze the time-varying characteristics of a nonstationary signal and is thus incorporated to evaluate the random error in the cavity ring-down spectroscopy (CRDS) experiments. With the numerical simulation of the influence of instabilities of sudden, slow, or periodic changes on the measurement accuracy in the ring-down process, DAVAR is proved to be an effective way to evaluate random error characteristics in an interfering environment. In order to minimize influences of time-varying noises in CRDS, a practical differential measurement method is proposed, in which wavelength modulation is applied to detect the ring-down times at the absorption peak and the nonabsorption peak in a timedivision manner. The validity of the differential measurement is proved with its ability to compensate the influence of the environment changes and improves the accuracy from $0.181 \mathrm{ppm}$ to $0.00914 \mathrm{ppm}$. The differential measurement method can be used to correct the time-varying error in real time and is helpful to improve the environmental adaptability of the CRDS instrument.

\section{Introduction}

As one of the widely accepted absorption spectroscopic technologies, cavity ring-down spectroscopy (CRDS) shows a rapid progress in company with the development of highquality mirrors and cavities. Two significant advantages bring CRDS with distinctive features different from other absorptive spectroscopic methods [1]. First, CRDS has ultrahigh spectral resolution due to its very narrow line width, which enables it to accurately distinguish the similar absorption wavelength of two different substances or the similar absorption wavelength of the same substance. Second, the usage of ultrahighly reflecting mirrors in constructing ring-down cavity enables an effective absorption path up to tens of kilometers and thus guarantees an extremely sensitive operation. CRDS was originally introduced to calibrate the reflectivity of ultrahighly reflecting mirrors. Since the pioneering works of O'Keefe and Deacon [2], this technique has been incorporated and thus played an important role in the detection of trace gas by virtue of its high detection sensitivity. Crosson used a CRDS-based analytical instrument to measure the concentration of greenhouse gases (i.e., $\mathrm{CH}_{4} / \mathrm{CO}_{2} / \mathrm{H}_{2} \mathrm{O}$ ) in the air, in an outfield environment, and conducted an evaluation on the long-term operational performance along with the U.S. National Oceanic and Atmospheric Administration (NOAA) [3]. Their work verifies the feasibility to use a CRDS-based apparatus for atmospheric monitoring. Chen et al. used the CRDS technology to measure methane and its isotopes. The CRDS spectrum analyzer of their work will be used for future Mars surface missions, since it is capable of meeting the detecting requirements of the Mars atmosphere relying on its extremely high sensitivity [4]. Chen et al. used the CRDS technology to detect $\mathrm{HCN}$ in oral exhalation, of which the sample concentration ranged from 0.9 to $10.9 \mathrm{ppb}$ [5]. HCN is considered as a potential marker for Pseudomonas aeruginosa lung infection. In addition to its application in the field of trace gas detection, the CRDS 
technology is also widely used in various fields, such as aerosol detection [6], combustion chemical analysis [7], and liquid chromatogram [8].

Error analysis plays an important role in determining CRDS measurement sensitivities. Huang and Lehmann studied the mode noises which arouse from high-order modes and suggested to insert spatial filtering apertures to suppress the formation of these high-order modes [9]. The authors also studied the beat-frequency noises from residual strains caused by linear birefringence and proposed suitable polarizing filtering means to improve the detection accuracy. Wang et al. studied the influences of white-noise amplitude on the fast Fourier transformation (FFT), discrete Fourier transformation, linear regression summation, Levenberg-Marquartt algorithm, and the least square method, respectively [10]. The authors carried out not only comparative analysis in terms of influences of noises, the accuracy and precision of fitting, and the fitting rate but also experimental studies as the verification of their discussion. Tan et al. studied the noises from mode mismatching, proposed an evaluation criterion based on the quality factor $Q$, and performed an analysis on the errors aroused from the inefficient intracavity build-up process [11]. It can be noted that these previous studies concern mainly on the error analysis in a single ring-down curve and discuss the timedomain characteristics of noises with standard Allan variance. For example, Stowasser et al. used Allan variance to perform a comparative analysis on the CRDS measurement accuracy, linearity, and drifts under different air pressures [12]. Huang and Lehmann used Allan variance to analyze the long-term stability of a CRDS apparatus and studied the relationship between mechanical deformations and the detection accuracy [13].

Although the standard Allan variance can easily fulfill the task of different error source identification and overall noise statistics derivation $[14,15]$, it is not able to characterize nonstationary, time-varying stochastic processes [16-18]. However, since the actual ring-down process is inevitably affected by the variations in the temperature, pressure, and even the performance change of different components, one can find that the standard Allan variance is no longer valid in the separation and characterization of these complex situations. Karhu et al. suggested that these time-varying errors were originated by the fluctuations in the cavity ring-down rate which were usually believed to be constant [19]. Another issue which needs to be noticed is that these time-varying drift noises in the ring-down rate present an ultimate limit for the sensitivity of CRDS, especially when long time averaging fitting is included. One possible solution is to record simultaneously the ring-down times at or away from the absorption, owing to the fact that the difference between them can be used directly to calculate the attenuation coefficient.

In this paper, the dynamic Allan variance (DAVAR) is used to simulate the feature of time-dependent noise and to analyze their influences on the CRDS measurement accuracy. In order to eliminate the influence of time-varying noise caused by environmental factors and instrument drift, this paper proposes a practical approach for differential measurement. By virtue of the detection of ring-down times with or without gas absorption, the error caused by timevarying noise is corrected. The validity of the above method was verified in the experiment by changing the temperature of the CRDS device.

\section{DAVAR and Simulation}

2.1. Allan Variance. It is supposed that the output signal of the cavity ring-down process is collected at a sampling period of $T_{0}$, and totally $N$ sets of curves are recorded in this paper. The ring-down time for the $i_{\text {th }}$ set $\tau_{i}$ (where $0<i \leq N$ ) is obtained by the CRDS formula [20] and fast fourier transform analysis method [21]. Separate $\tau_{i}$ into $K=N / M$ groups with $M \leq(N-1) / 2$ representing the number of data within each group. Then, the average of each group can be given by

$$
\overline{\tau_{k}}(M)=\frac{1}{M} \sum_{j=1}^{M} \tau_{(k-1) M+j}, \quad k=1,2, \ldots, K^{\sigma},
$$

where $\sigma$ is a parameter in connection with the Allan variance.

According to the definition of the Allan variance,

$$
\begin{aligned}
\sigma^{2}(T) & =\frac{1}{2}\left\langle\left[\bar{\tau}_{k+1}(T)-\bar{\tau}_{k}(T)\right]^{2}\right\rangle \\
& =\frac{1}{2\left(K^{\sigma}-1\right)} \sum_{k=1}^{K^{\sigma}-1}\left[\bar{\tau}_{k+1}(T)-\bar{\tau}_{k}(T)\right]^{2},
\end{aligned}
$$

where $T=T_{0} \cdot M$ is the correlation time of each group, the triangular bracket denotes an overall average, and $\tau_{i}(M)$ is a discrete sequence. The curve showing the dependence of $\sigma^{2}(T)$ on $T$ can be obtained by adjusting the quantity of the correlation time $T$. A further double logarithmic treatment of the curve $\sigma^{2}(T) \sim T$ gives then the Allan variance.

2.2. DAVAR. As a method proposed by Galleani et al., the DAVAR was originally used in the time-varying stability measurement of an atomic clock [16-18]. DAVAR characterizes a time-varying signal $\tau(t)$ and obtains its Allan variance through sliding estimation of the signal sequence with different time periods. DAVAR thus introduces the temporal information into the standard Allan variance, which may be expressed in the form of a three-dimensional graph characterizing the statistical evaluation tendency of the noises over time.

Assume that $\tau(t)$ is the discrete CRDS signal sampled and recorded with a length of $N$. A sliding interception on $\tau(t)$ with a window function $P_{w}(t)$ centered at the point $T_{c}$ of the length $L_{w}(t)$ gives the intercepted signal as follows:

$$
\begin{gathered}
\tau\left(t, T_{c}\right)=\tau(t) P_{w}(t), \\
t-\frac{L_{w}}{2} \leq T_{c} \leq t+\frac{L_{w}}{2} .
\end{gathered}
$$

There are $L_{w}$ continuous data $\tau\left(t, T_{0}\right)$ intercepted at the sampling period of $T_{0}$. Follow the definition of Allan 
variation and separate $\tau\left(t, T_{0}\right)$ into groups with $M$ continuous data. $T=T_{0} \cdot M$ gives the correlation time within the group. Let $T$ takes the values of $T_{0}, 2 T_{0}, \ldots, M T_{0}$, and the average within each correlation time can be expressed as

$$
\overline{\tau_{k}}(t, T)=\frac{1}{M} \sum_{j=1}^{M} \tau_{(k-1) M+j}, \quad k=1,2, \ldots, L_{W}-M+1,
$$

where $\bar{\tau}_{k}(t, T)$ is the average of the array within the correlation time starting from the $k^{\text {th }}$ data of the intercepted $L_{w}$ continuous data. Totally, $L_{w}-M+1$ arrays can be obtained with a step length of $M$ within the data length of $L_{w}$. The average difference between the two neighboring arrays is

$$
\Delta \tau_{k, k-1}=\overline{\tau_{k+1}}(t, T)-\overline{\tau_{k}}(t, T) .
$$

Group all the average difference values between every two neighboring arrays within $L_{w}$ and thus form a set including $L_{w}-M$ elements. According to the definition of Allan variance, one has

$$
\begin{aligned}
\sigma^{2}(t, T) & =\frac{1}{2}\left\langle\left[\bar{\tau}_{k+1}(t, T)-\bar{\tau}_{k}(t, T)\right]^{2}\right\rangle \\
& =\frac{1}{2\left(L_{W}-M-1\right)} \sum_{k=1}^{L_{W}-M-1}\left[\bar{\tau}_{k+1}(t, T)-\bar{\tau}_{k}(t, T)\right]^{2} .
\end{aligned}
$$

2.3. Simulation. In case that the CRDS apparatus works in an unsatisfactory environments, the changing temperature, pressure, or vibration situation lead to the misalignment of the cavity or to the variation of the absorption cross section of the trace gas. This results in the deterioration in the measurement accuracy. In order to simulate the actual situation of unstable environments such as periodic variation, sudden change, and slow evolution, additional noises are imposed on the ring-down time sequence in the present paper, and the influences on the measurement accuracy are analyzed with standard Allan variance and DAVAR.

Gaussian noise as an additive noise exists in the decaying time series. In this paper, the amplitude of Gaussian noise is modulated sinusoidally to simulate the influence of environment. $\tau(t)$ is a ring-down time sequences with a length of 8000 data. The mean of $\tau(t)$ is $36 \mu$ s. The Gaussian noise intensity is $40 \mathrm{~dB}$. A sinusoidally modulated Gaussian noise of $10 \mathrm{~dB}$ is then added to $\tau(t)$, as shown in Figure 1(a). Allan variance is calculated as the difference between the average value of the sampled data in the previous correlation time and the next correlation time. If the average value of the data in the correlation time does not change, the effect of change cannot be exhibited. Since the mean value of the Gaussian noise of the sinusoidal modulation tends to 0 , the analysis using Allan variance cannot reflect the influence of the sinusoidal modulation noise. The trends of the two curves are basically the same. The noise equivalent absorption coefficient has changed from $9.8 \times 10^{-9} \mathrm{~cm}^{-1} \cdot \mathrm{Hz}^{-1 / 2}$ to $1.5 \times 10^{-8} \mathrm{~cm}^{-1} \cdot \mathrm{Hz}^{-1 / 2}$. The difference in absorption coefficient is due to the short correlation time setting. In order to increase the time resolution, the correlation time in the Allan variance is set relatively small. This will cause the mean value of noise loaded during the relevant time may not be zero, which will increase the equivalent noise figure. Using DAVAR to analyze the loaded sinusoidal modulation noise, as shown in Figure 1(c), in the time period unaffected by noise, such as from $0 \mathrm{~s}$ to $2000 \mathrm{~s}$ and $6000 \mathrm{~s}$ to $8000 \mathrm{~s}$, the noise equivalent absorption coefficient is about $4.4 \times 10^{-9} \mathrm{~cm}^{-1} \cdot \mathrm{Hz}^{-1 / 2}$. In the period of $2000 \mathrm{~s}-6000 \mathrm{~s}$ affected by noise, the noise equivalent absorption coefficient changes from $4.4 \times 10^{-9} \mathrm{~cm}^{-1} \cdot \mathrm{Hz}^{-1 / 2}$ to $2.2 \times 10^{-8} \mathrm{~cm}^{-1} \cdot \mathrm{Hz}^{-1 / 2}$, and the change shows a sine wave trend, which is consistent with the loaded noise.

Changes in gas pressure and environmental vibrations may cause sudden changes in the ring-down time. The sudden-changed signal is a discontinuous change, which usually indicates the change of the operating state of the instrument, which may be caused by gas pressure shock or vibration to cause the resonance cavity to detune, or it may be a circuit failure. A $2 \mu$ s mutation signal was added to the decay time series, in which ring-down time is $36 \mu$ s and the Gaussian noise intensity is $40 \mathrm{~dB}$. Allan variance was used to analyze the change of the stagnation time series before and after the sudden-changed signal was added, as shown in Figure 2(b). The noise equivalent absorption coefficient is $9.1 \times 10^{-7} \mathrm{~cm}^{-1} \mathrm{~Hz}^{-1 / 2}$. It can be known that the large variance caused by the sudden-changed signal makes the calculation divergent, and subsequent sampling cannot be analyzed. Allan variance can reflect the change of the signal, and it is impossible to obtain the moment when the abrupt signal occurs and the impact on the entire process. Figure 2(c) uses DAVAR to analyze the time series containing sudden-changed signals. The noise equivalent coefficient before the sudden change is $4.6 \times 10^{-9} \mathrm{~cm}^{-1} \cdot \mathrm{Hz}^{-1 / 2}$, and the NEA within the duration of the sudden change signal is $1.4 \times 10^{-8} \mathrm{~cm}^{-1} \cdot \mathrm{Hz}^{-1 / 2}$. DAVAR has the resolution capability in the time domain, which can track the dynamic change process of the abrupt signal in time. It is also noticed that the ability of real-time tracking and reliability of performance estimation is strongly related with the type and length of the configured calculation window [22-24]. The window type in this article is a rectangular window with a length of 100 samples.

Drift noise is to simulate the temperature drift of a device in CRDS for a short time or the degradation of device performance for a long time. Add drift noise to the $40 \mathrm{~dB}$ Gaussian noise decay time series. Noise drift is twice the standard deviation of Gaussian noise, as shown in Figure 3(a). It can be known from formula (2) that when the average value of the signal changes during the correlation time, the Allan variance can reflect this change, as shown in Figure 3(b). Drift noise prevents repeated sampling from improving detection accuracy, and the minimum equivalent noise absorption coefficient is $1.3 \times 10^{-9} \mathrm{~cm}^{-1}$. DAVAR can show the change of drift noise in the time domain, as shown in Figure 3(c), the noise equivalent absorption coefficient changed from $4.5 \times 10^{-9} \mathrm{~cm}^{-1} \cdot \mathrm{Hz}^{-1 / 2}$ to $8.4 \times 10^{-9} \mathrm{~cm}^{-1} \cdot \mathrm{Hz}^{-1 / 2}$. 


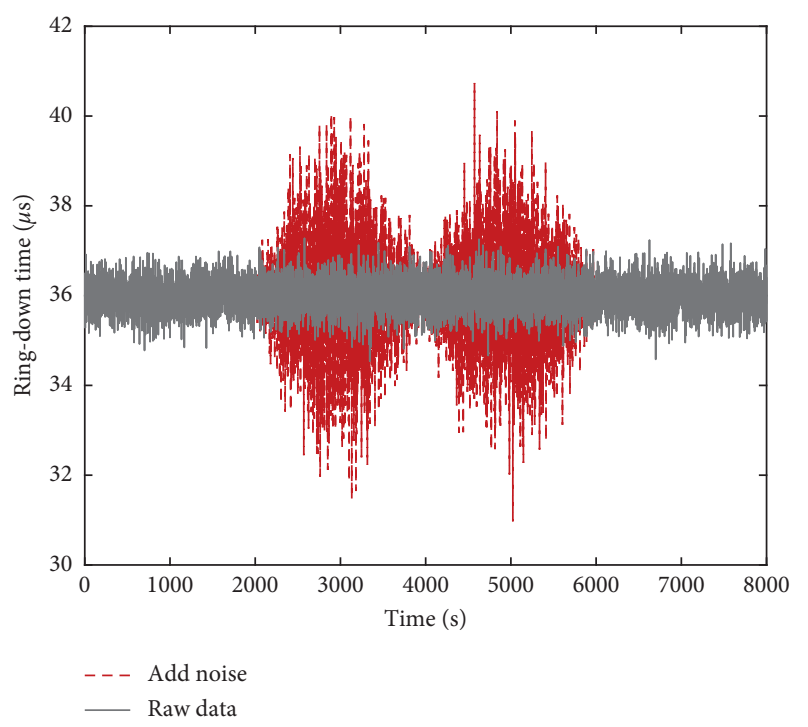

(a)

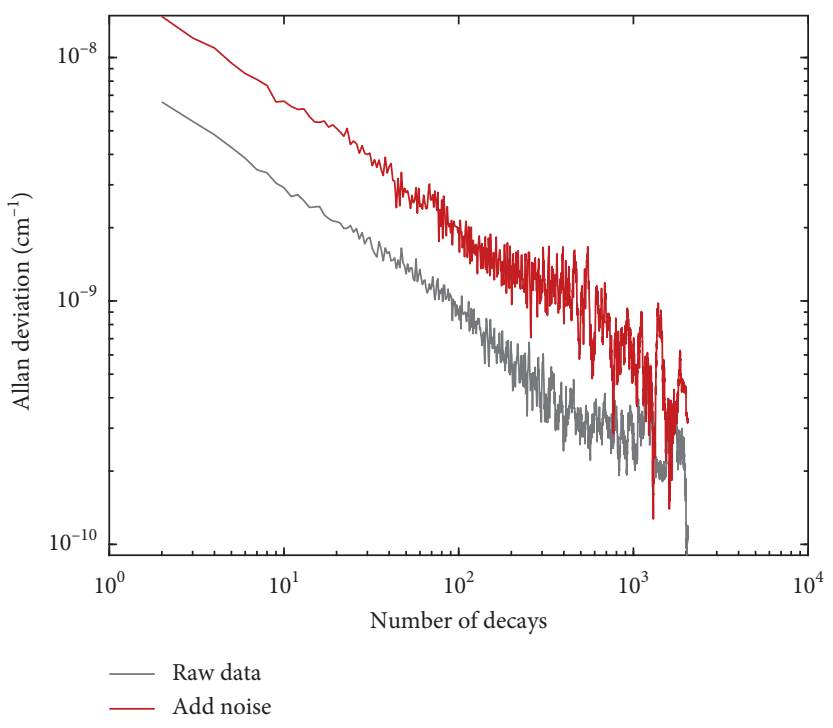

(b)

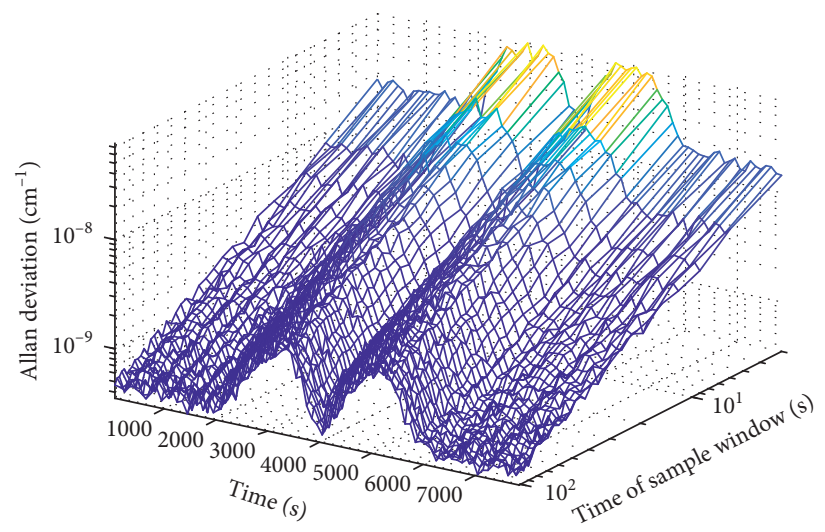

(c)

FIGURE 1: Allan variance and DAVAR analysis of modulated signals. (a) Add sinusoidally modulated Gaussian noise to the ring-down time series. (b) Using Allan variance to analyze the effect of noise on detection accuracy. (c) DAVAR analysis detection accuracy changes over time.

During the detection process, the CRDS device is affected by the environment and its own factors, and the ring-down time will change in the time domain, such as sudden changes, drifts, and periodic changes. According to the above simulation and analysis, Allan variance cannot analyze nonstationary time-varying signals. However, DAVAR is able to clearly and intuitively reveal the temporal evolution of the signal variance by tracking the error changes via a sliding window. DAVAR gives thus an effective approach to evaluate the time-varying characteristics of random errors in CRDS.

\section{Experiment and Analysis}

3.1. Experiment and Analysis of Temperature Variation in a Ring-Down Cavity. A continuous wave CRDS (CW-CRDS) experiment is constructed to detect the concentration of methane $\left(\mathrm{CH}_{4}\right)$, as sketched in Figure 4. A fiber-coupled output distributed feedback diode laser (DFB-LD, NLK1U5FAAA, NEL) with the center wavelength of $\sim 1654 \mathrm{~nm}$ is used as the light source. After passing through a set of mode-matching optics, the infrared laser beam is coupled into the $360 \mathrm{~mm}$-long linear ring-down cavity which is composed of two identical concave high reflectivity mirrors. Each mirror has a radius of curvature of $500 \mathrm{~mm}$ and a reflectance higher than 0.9999 around the ring-down wavelength. An optical isolator (IO-4-1650-VLP, Thorlabs) is inserted before the coupling optics in order to suppress the optical feedback caused by the optics. A laser controller (LDC-3724C, Lightwave) is used to set the operation temperature and modulating current of the DFB laser so as to adjust the output wavelength of the laser. The output wavelength of the DFB laser is then adjusted to $1653.722 \mathrm{~nm}$, i.e., the $2 \mathrm{v} 3$ absorption band of methane. With a periodic triangular wave signal at the frequency of $10 \mathrm{~Hz}$ from the signal generator (33600A, Aglient), the DFB laser achieves a spectral scanning region slightly larger than the free spectral range (FSR) of the ring-down cavity and thus guarantees the happening of laser-cavity spectral matching. The transmission from the ring-down cavity is then collected by a photodetector (PDA10CS-ES, Thorlabs) with a focusing lens. 


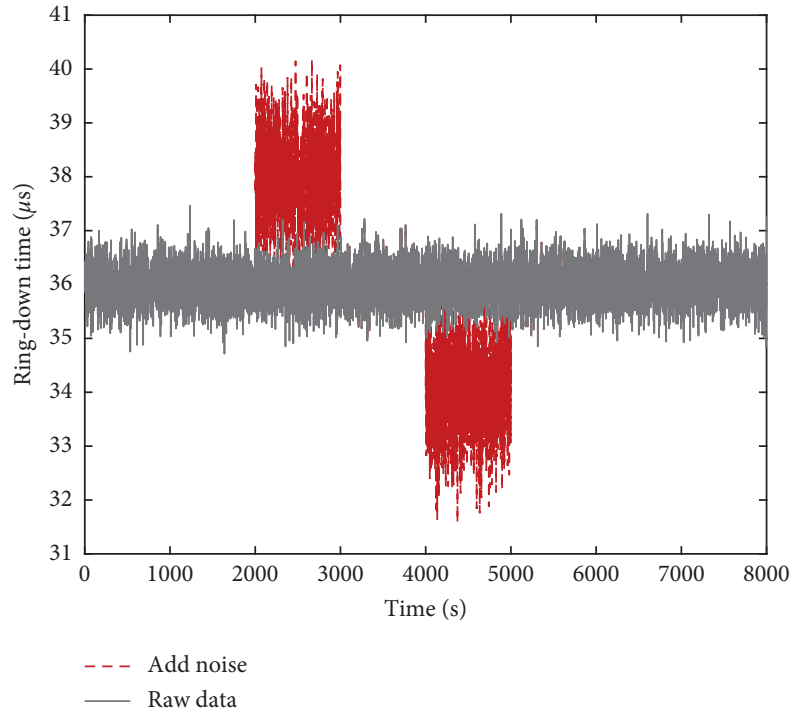

(a)

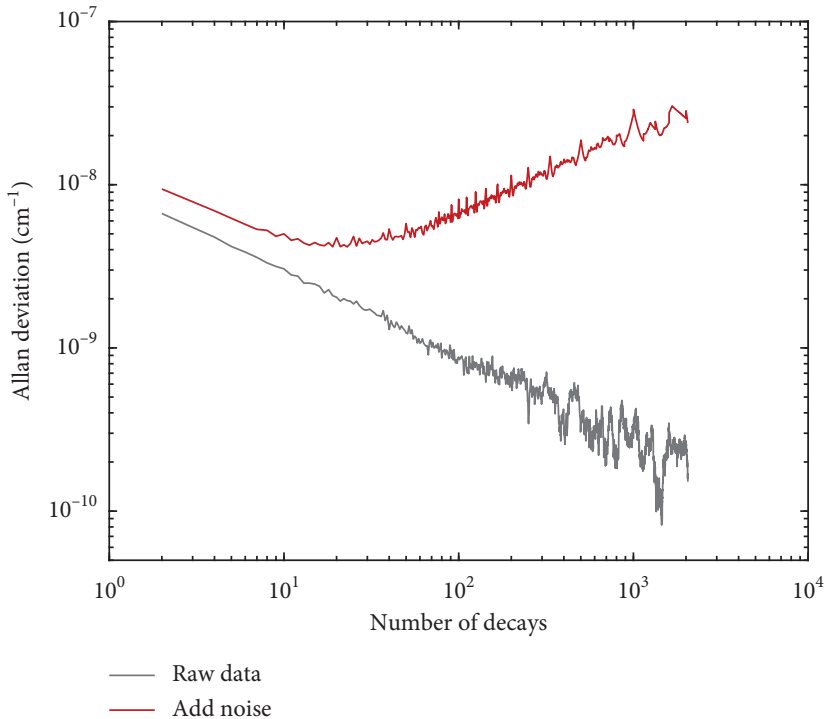

(b)

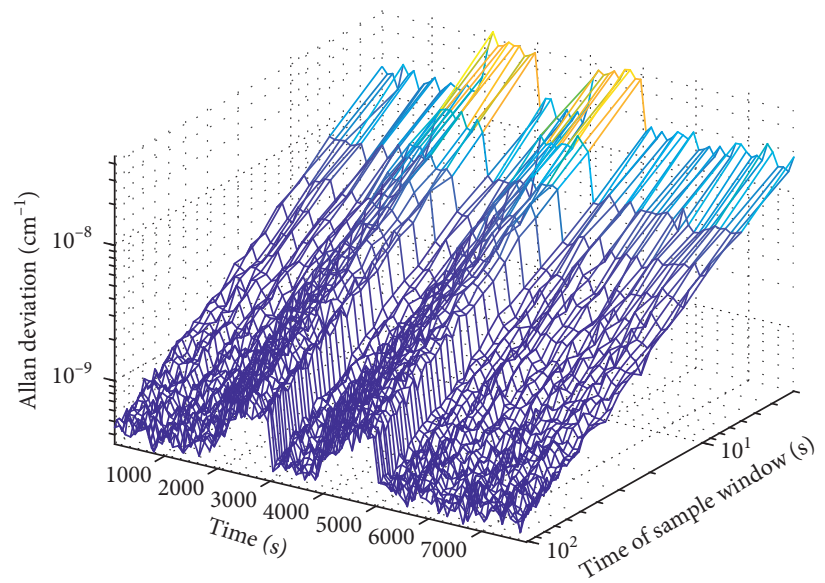

(c)

FIGURE 2: Allan variance and DAVAR analysis of sudden-changed signals. (a) Add sudden noise to ring-down time series. (b) Using Allan variance to analyze the effect of noise on detection accuracy. (c) DAVAR analysis detection accuracy changes over time.

When the output of the photodetector reaches the preset threshold, an oscilloscope (MSO 4104C, Tektronix) is triggered on and sends out an order to sharply turn off the modulation from the signal generator. Thus, it enables a laser-cavity mismatching shutoff and completes the whole ring-down process. In the experiment, the sample gas enters the cavity through a mass flow controller, and gas pressure in the cavity is 0.996 bar. Additional desiccant and particle filter are introduced on both ends of the cavity to stop the entrance of water vapor and fine particles.

A thermistor placed within the cavity housing measures the temperature. A thermal controller (TCM-X107, Chengdu Yexian Technology) functions to receive the information from the thermistor and precisely control the temperature of the ring-down cavity with a thermo-electric cooler (TEC) fixed at the center of the aluminum alloy plate. The first step of the experiment is to set the temperature of the ring-down cavity at $25^{\circ} \mathrm{C}$ and measure the cavity ringdown time of high-purity nitrogen gas $\left(\mathrm{N}_{2}\right.$ with a purity of
99.99\%). Repeat the measurement and achieve 8000 ringdown events. The ring-down times for different events are extracted with fast fourier transform analysis method as shown in Figure 5. The result is then analyzed with Allan variance which gives the noise equivalent absorption coefficient of $2.4 \times 10^{-10} \mathrm{~cm}^{-1}$.

The experiment goes on by continuously introducing the high-purity nitrogen gas into the cavity but slowly raises the temperature of the ring-down cavity to $28^{\circ} \mathrm{C}$ at a rate of $0.001^{\circ} \mathrm{C} / \mathrm{s}$. The rate is selected as the experiment tells that only a slow variation in the thermal situation guarantees no violent sudden change to the cavity, and thus no failure in ring-down signal collection happens. A thermal controller used in the experiment recorded the temperature change process of the bottom plate, as shown in Figure 6(a). Temperature stability at $25^{\circ} \mathrm{C}$ is $0.0036^{\circ} \mathrm{C}$, and temperature stability at $28^{\circ} \mathrm{C}$ is $0.013^{\circ} \mathrm{C}$. The decay time during the temperature change collected using the CRDS experimental device is shown in Figure 6(b). When the cavity temperature 


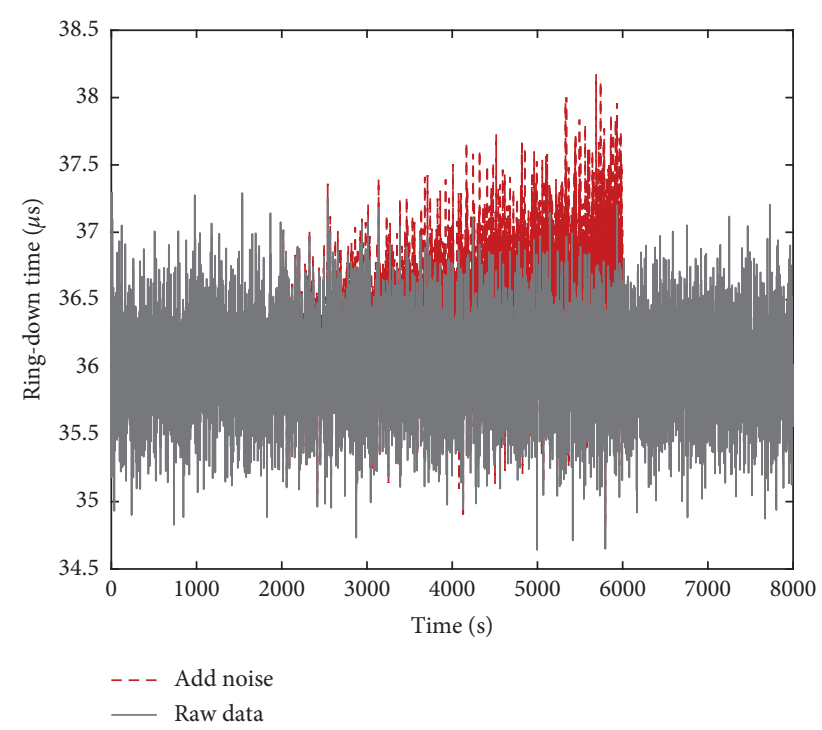

(a)

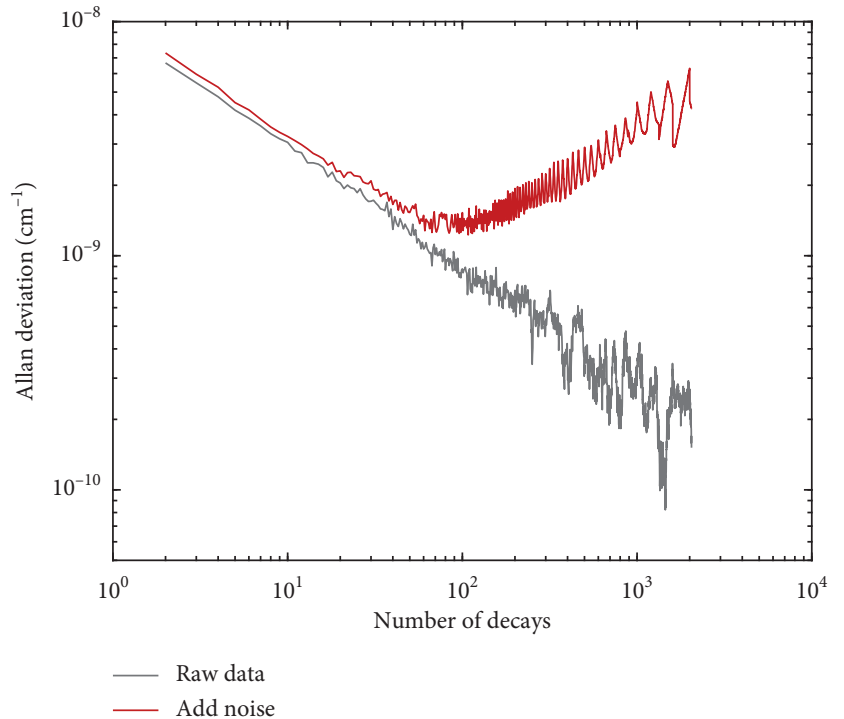

(b)

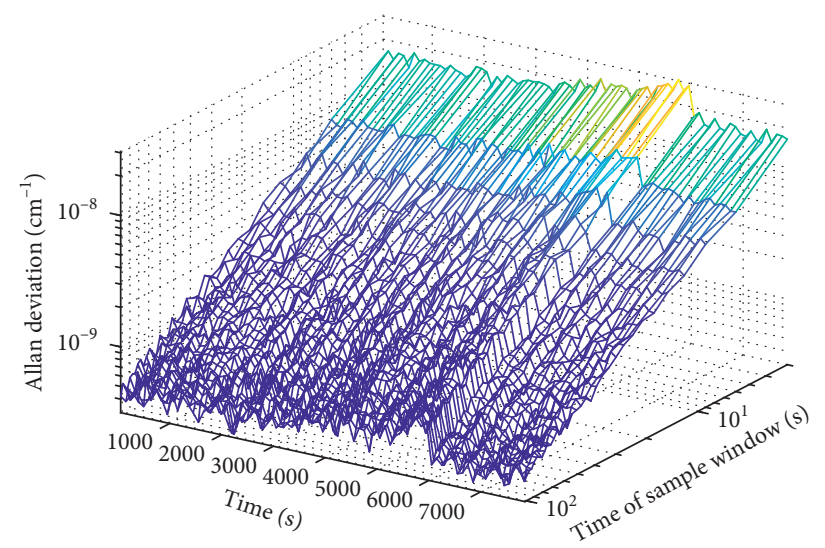

(c)

Figure 3: Allan variance and DAVAR analysis of slow-changed signals. (a) Add slow change noise to ring-down time series. (b) Using Allan variance to analyze the effect of noise on detection accuracy. (c) DAVAR analysis detection accuracy changes over time.

is $25^{\circ} \mathrm{C}$, the mean of ring-down time is about $35.7 \mu \mathrm{s}$, and when the temperature changes to $28^{\circ} \mathrm{C}$, the mean of ringdown time decays to $30.6 \mu \mathrm{s}$.

The above experiment was divided into three stages according to the temperature, $25^{\circ} \mathrm{C}$, temperature change process, and $28^{\circ} \mathrm{C}$. Allan variance and DAVAR were used to analyze the experimental data. According to the analysis of Allan variance, the minimum noise equivalent absorption coefficient is $1.6 \times 10^{-8} \mathrm{~cm}^{-1}$, and the time normalized noise equivalent absorption coefficient is $1.1 \times 10^{-8} \mathrm{~cm}^{-1} \cdot \mathrm{Hz}^{-1 / 2}$. Due to the temperature change process, the mean of the samples in the adjacent correlation time changes, making the Allan variance unable to evaluate the entire acquisition process. The DAVAR is used to analyze the experimental data, as shown in Figure 7(b). The noise equivalent absorption coefficient is $1.5 \times 10^{-9} \mathrm{~cm}^{-1} \cdot \mathrm{Hz}^{-1 / 2}$ at a temperature of $25^{\circ} \mathrm{C}$, and it is basically consistent with Allan analysis of variance. Due to the temperature change process, the noise equivalent absorption coefficient increased to $7.9 \times 10^{-9} \mathrm{~cm}^{-1} \cdot \mathrm{Hz}^{-1 / 2}$. After the temperature stabilized at $28^{\circ} \mathrm{C}$, the noise equivalent absorption coefficient is $3.4 \times 10^{-9} \mathrm{~cm}^{-1} \cdot \mathrm{Hz}^{-1 / 2}$. The reason for noise equivalent absorption coefficient enlargement is that the stability of cavity temperature becomes worse. The DAVAR analysis clearly shows the three stages of temperature change and reflects the effect of temperature control accuracy on the stability of the decay time.

In the experiment, the ring-down cavity is fixed on a plate made of aluminum alloy with a thermal expansion coefficient of $23.6 \mu \mathrm{m} / \mathrm{m} \cdot \mathrm{K}$. As compared with the material of invar steel with a thermal expansion coefficient of $1.6 \mu \mathrm{m} /$ $\mathrm{m} \cdot \mathrm{K}$, aluminum alloy shows a larger deformation under thermal changing environment. With a conventional finite element analysis, as shown in Figure 8, one can notice that when the temperature of the aluminum alloy base plate changes by $3^{\circ} \mathrm{C}$, the base plate for fixing the ring-down cavity of mirror is deformed in $87.2 \mathrm{~nm}$. This deformation due to temperature produces an off-axis angle of 1.3 arc-second with the optical axis. The generation of the off-axis angle will increase the loss in the cavity and shorten the ring-down time. The off-axis angle increases the loss in the cavity and 


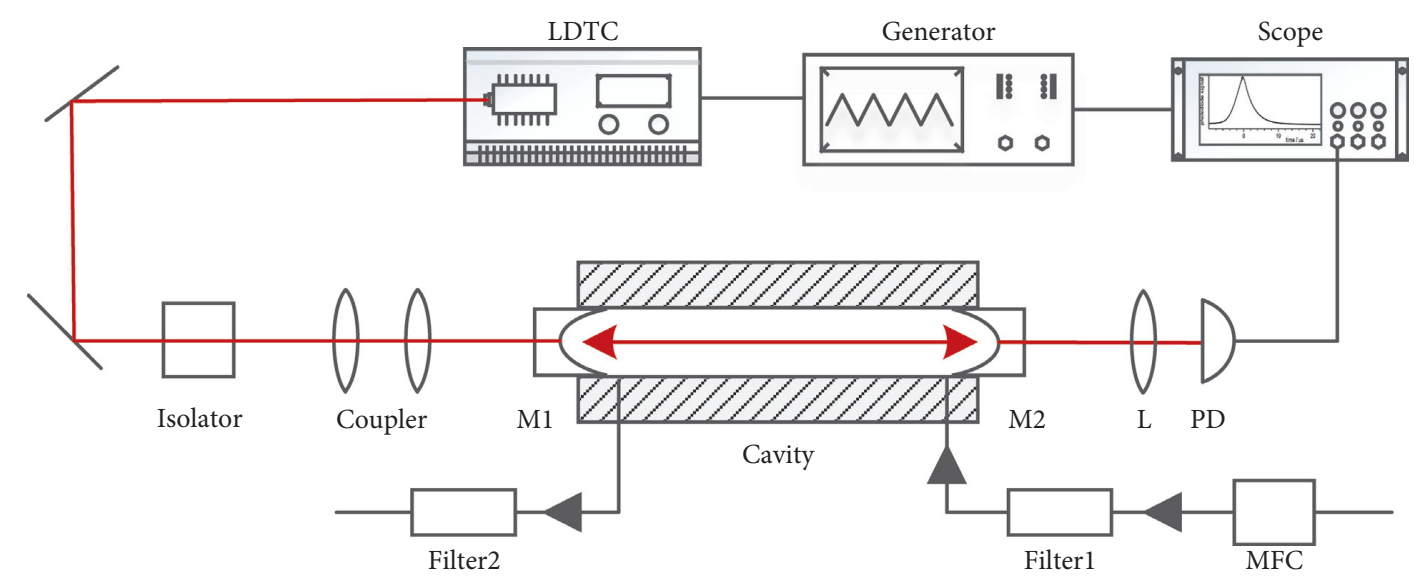

Figure 4: Schematic diagram of the experimental device. LDTC, laser driver and temperature controller. Generator, signal generator. M1 and M2, the RDC mirrors. RDC, ring-down cavity. L, focusing lens. PD, photodetector. MFC, mass flow controller.

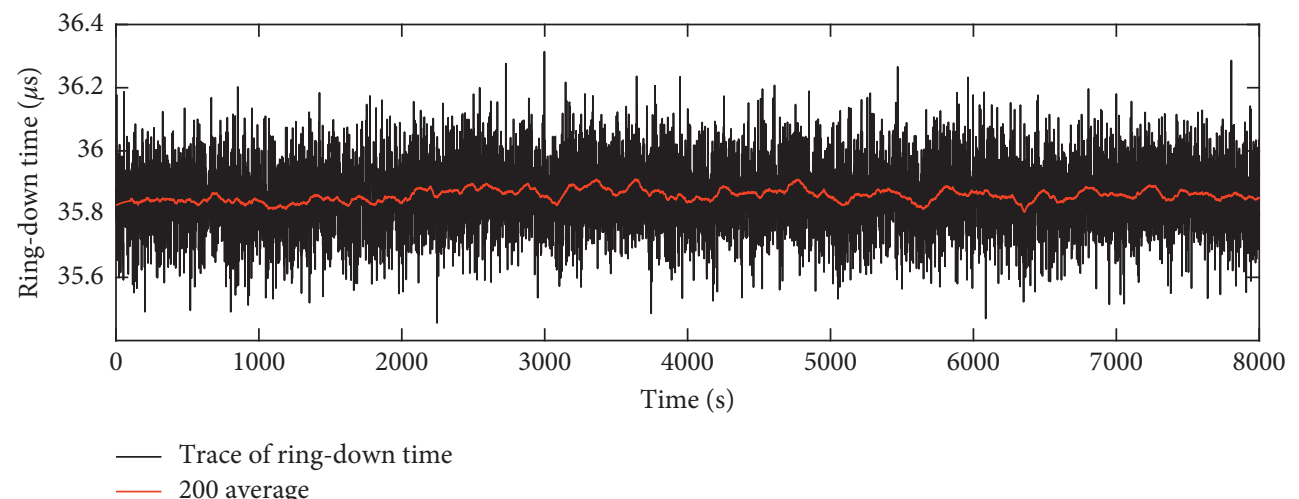

Figure 5: The 8000 sets of cavity ring-down time with $\mathrm{N}_{2}$ at $25^{\circ} \mathrm{C}$.

shortens the ring-down time. The aluminum alloy base plate used in the experiment amplifies this phenomenon due to its large thermal expansion coefficient. Repeating the above finite element analysis process, replacing the aluminum alloy material with invar steel, the base plate has a deformation of about $5.7 \mathrm{~nm}$, and the off-axis angle is about 0.17 arc seconds. The influence of temperature changes cannot be eliminated by using the invar steel base plate.

\subsection{Differential Measurement Method and Experiment.} Both the temperature of the ring-down cavity and the pressure of the input gas are always strictly controlled in CRDS devices, to the level of a few hundredths of a $K(1 \sigma)$ and pressure accuracy of 0.05 Torr, respectively, as in the product fabricated by the Picarro Inc. [3]. This point is quite similar to the fact that always a very accurate reference of GROUND is defined in electronics. The existence of this reference helps to perform the measurement by merely the detection of the relative values of a single-ended signal with respect to GROUND. Therefore, the accuracy of the measured signal depends on the consistency of this "GROUND" in the system during the whole measurement. When this reference fluctuates due to influences of the external environment, the error caused by such fluctuations is also superimposed on the results. In CRDS, the ring-down time $\tau_{0}$ can be regarded corresponding to the abovementioned "GROUND" in the electrical measurements.

Using the differential method for suppressing common mode interferences in electrical measurements for reference, researchers have implemented differential measurements by measuring the "GROUND." Courtois et al. [25] and Ye and Hall [26] use acousto-optic modulators as a frequency shifter to fine-tune the laser frequency and measure the ring-down time of the absorption peak and the nonabsorption peak in a time-sharing manner to realize differential measurement. In their experiments, the output frequency of the laser is fixed, and two serial or parallel acousto-optic modulators are used to modulate the output frequency. The differential measurement may also be implemented using two lasers [13]. One of the two lasers has a wavelength corresponding to an absorption peak of the gas to measure absorption loss, and the other is used to measure the drift of the instrument, which is to be corrected by the differential measurement method.

Based on the analysis of existing methods and drawing on the principle of differential measurement in electrical measurement, this paper proposes a practical differential measurement method. By modulating the output frequency of a laser quickly, the ring-down times at the absorption and nonabsorption peaks are measured in a time-sharing 


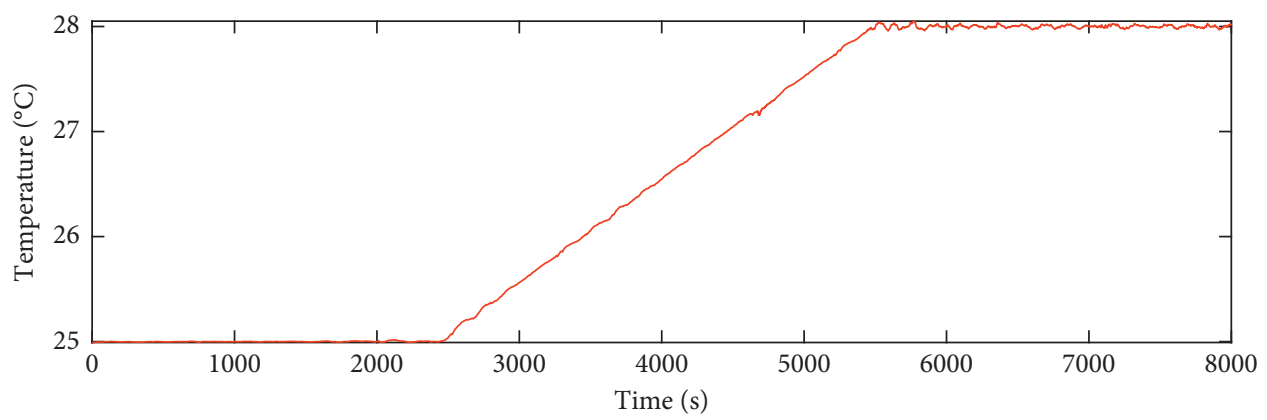

(a)

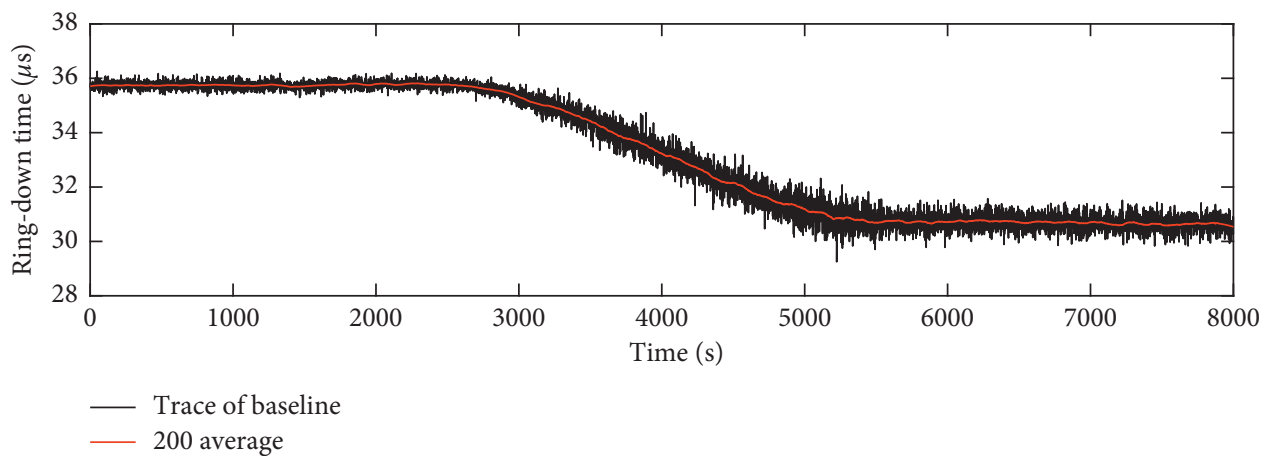

(b)

FIgURE 6: The variation of ring-down time with temperature. (a) Change of cavity temperature recorded by thermal controller. (b) Ringdown time at different temperatures.

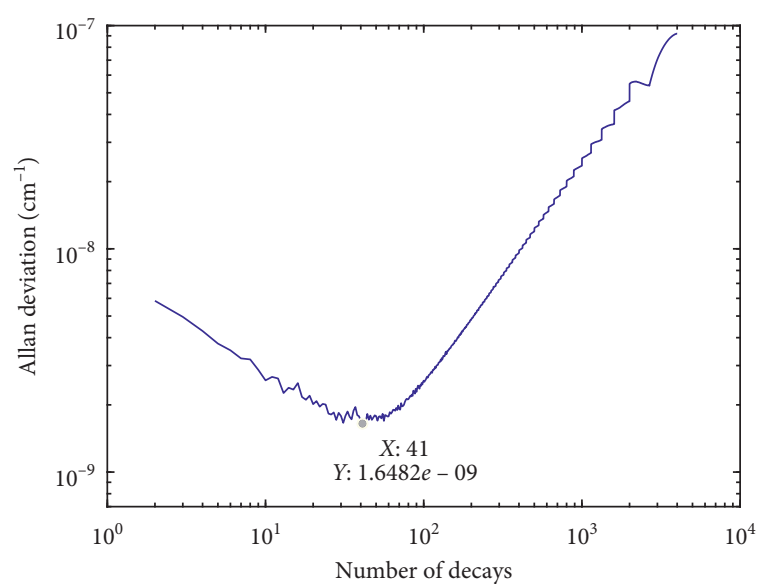

(a)

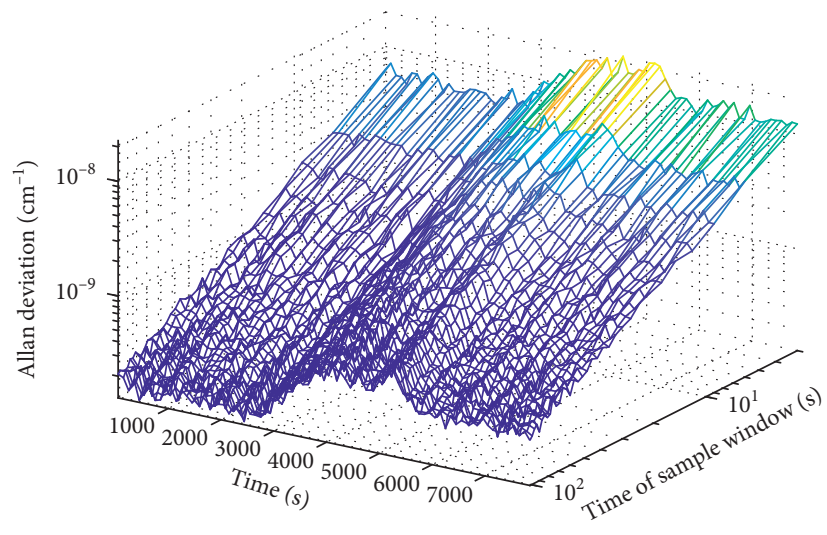

(b)

FIGURE 7: Comparative analysis of Allan variance and DAVAR. (a) The relationship between ring-down time and temperature change in Allan variance analysis. (b) The relationship between ring-down time and temperature change in DAVAR.

manner. The ring-down time at the nonabsorption peak is used to record the influence of the instrument on the environment and its drift. At the absorption peak, the ringdown time becomes shorter due to gas absorption loss. The gas concentration is calculated by the change of the ringdown time. The measurement at the absorption peak is also affected by environmental factors and instrument drift.

In this paper, a self-developed laser driving circuit is used instead of the LDTC in the experimental device of
Figure 4. The steps for differential detection are as follows:

(1) Set the base voltage $V_{\mathrm{OFF}}$ so that the laser output wavelength is at the nonabsorption peak

(2) Load the modulating waveform on the MODULATE terminal, and the wavelength can be matched with the cavity mode by laser current scanning 


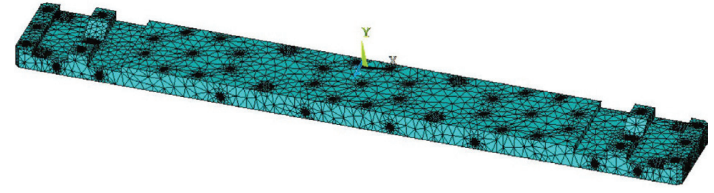

(a)

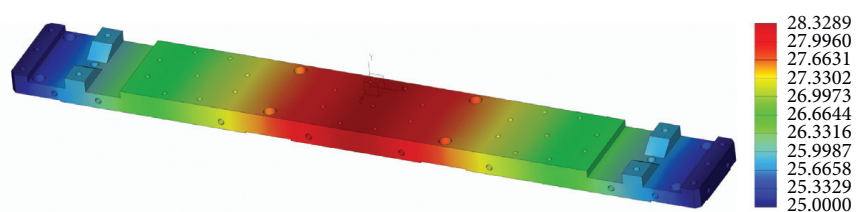

(b)

Figure 8: Thermal deformation finite element analysis of the ring-down cavity. (a) Finite element meshing of the base plate. (b) Temperature distribution of the bottom plate when the temperature changes to $28^{\circ} \mathrm{C}\left(25^{\circ} \mathrm{C}\right.$ at room temperature).

(3) When the amplitude of the signal received by the detector reaches the threshold, SHUTOFF realizes the laser shutdown process by detuning

(4) Set the base voltage $V_{\mathrm{ON}}$ so that the laser output wavelength is at the absorption peak; repeat steps 2 and 3 and acquire the ring-down time at the absorption peak

A laser spectrum analyzer (771B-NIR, Bristol instruments Inc.) was used to test the laser output wavelength stability, and the output stability within 1 hour was $\pm 1.2 \mathrm{pm}$, which was consistent with the performance of LDTC in Figure 4. The large current modulation will affect the thermal stability of the laser. The difference between the voltage settings of $V_{\mathrm{ON}}$ and $V_{\text {OFF }}$ should be as small as possible.

By setting the parameters of $V_{\mathrm{ON}}$ and $V_{\mathrm{OFF}}$, the wavelength variation is about $0.4 \mathrm{~nm}$, which can be seen from Figure 9. MODULATE is loaded with a $20 \mathrm{~Hz}$ triangle wave. Due to the symmetry of the triangle wave signal, it can achieve $40 \mathrm{~Hz}$ detection. The free spectral range of the cavity is $441 \mathrm{MHz}$, and the wavelength modulation range is set to $4 \mathrm{pm}$.

Then, methane standard gas ( $1 \mathrm{ppm}$, uncertainty is $2 \%$ ) is injected into the cavity. When the temperature of the ringdown cavity changes from $25^{\circ} \mathrm{C}$ to $28^{\circ} \mathrm{C}$, the differential measurement method detects the ring-down time at the absorption peak and the nonabsorption peak, as shown in Figure 10. In order to ensure the stability of the wavelength during the switching process, the detection frequency is also set to $1 \mathrm{~Hz}$. Due to the influence of temperature change, the decay time at the absorption peak has changed from $21.1 \mu \mathrm{s}$ to $19.0 \mu \mathrm{s}$. The Pearson correlation coefficient is introduced to evaluate the relationship between the ring-down time at the absorption peak and the nonabsorption peak [27]. The Pearson correlation coefficient between the ring-down time at the absorption peak and the nonabsorption peak detected by the differential method is 0.95 . Due to the effects of temperature changes, two independent detections show a very high correlation. The ring-down time series $\tau$ at the absorption peak is affected by the gas absorption attenuation, and it is also affected by the drift of instrument detection baseline due to temperature change.

The theory of CRDS gives that the concentration of trace gas can be expressed as [28]

$$
N=\frac{1}{c \sigma(\nu)}\left(\frac{1}{\tau}-\frac{1}{\tau_{0}}\right)
$$

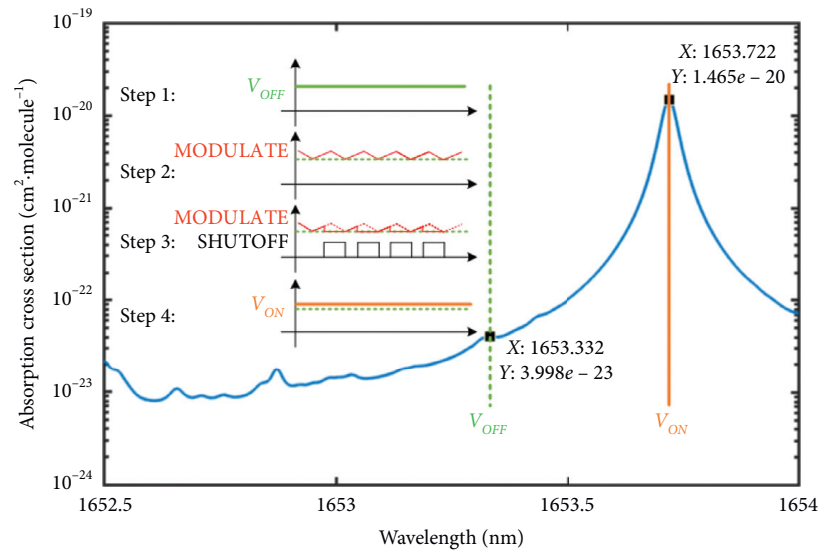

FIGURE 9: Schematic diagram of operation process of differential measurement.

where $c$ is the light speed, $\sigma(v)$ the absorption cross section, $\tau$ the ring-down time when the input laser wavelength coincides with the strongest absorption in the absorption spectrum, and $\tau_{0}$ the ring-down time of the empty cavity when no absorption exists. According to the database of HITRAN, the gas absorption cross section is related to parameters such as temperature and pressure [29]. In this paper, the absorption cross sections at different temperatures are calculated using the Voigt linear [30] and the HIRAN database. The absorption cross section at $25^{\circ} \mathrm{C}$ is $1.479 \times 10^{-20} \mathrm{~cm}^{2}$ molecule ${ }^{-1}$, and the absorption cross section at $28^{\circ} \mathrm{C}$ is $1.471 \times 10^{-20} \mathrm{~cm}^{2} \cdot$ molecule ${ }^{-1}$. The detailed calculation process is explained in the Appendix. Therefore, during the experiment from $25^{\circ} \mathrm{C}$ to $28^{\circ} \mathrm{C}$, the differences in absorption cross sections were ignored.

According to formula (7), cavity decay spectroscopy is self-calibrated. By substituting $\tau$ and $\tau_{0}$ obtained by differential measurement into formula (7), the effect on temperature change can be corrected. In this paper, the concentration of standard methane gas was calculated using the experimental device shown in Figure 4. The ring-down time shown in Figure 5 was used as the ring-down time at the nonabsorption peak. The calculated result is shown in Figure 11(a) with the standard deviation of $0.181 \mathrm{ppm}$. The test results using the differential measurement are shown in Figure 11(b), and the standard deviation is $0.00914 \mathrm{ppm}$. The effect of temperature change is corrected in real time by differential measurement, and the detection accuracy is improved about 19 times. 


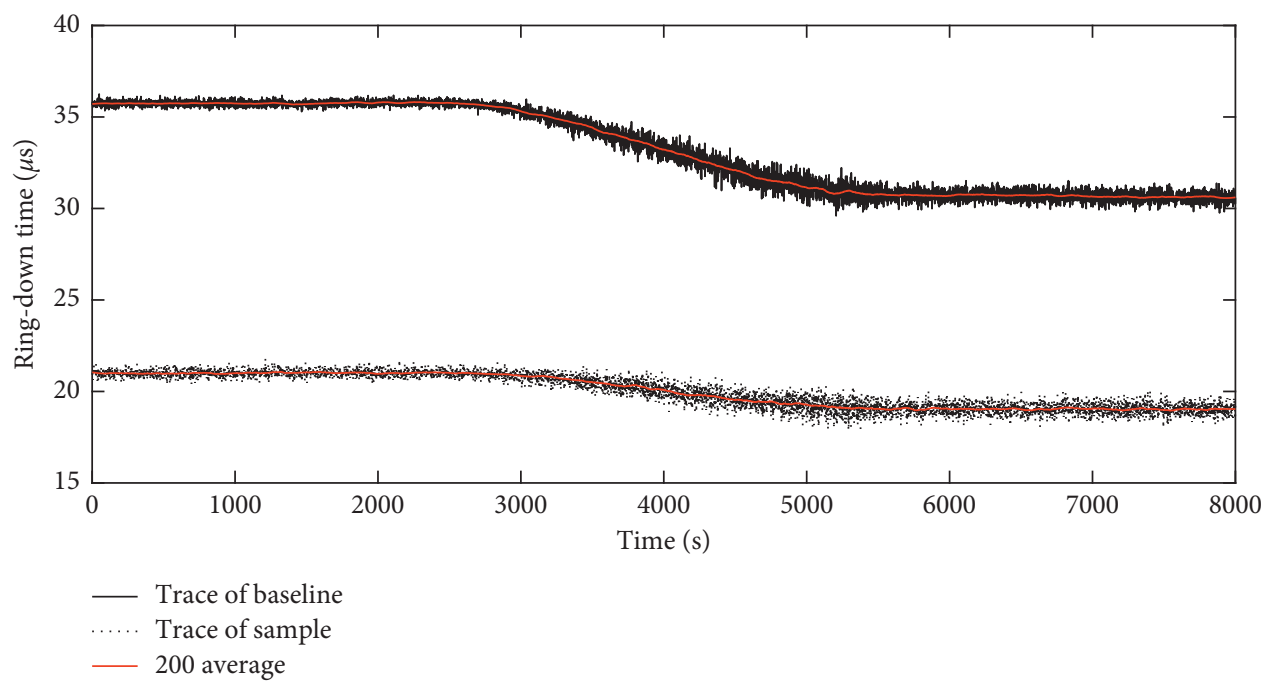

FIGURE 10: The ring-down time $\tau$ and $\tau_{0}$ acquired by differential measurement method.

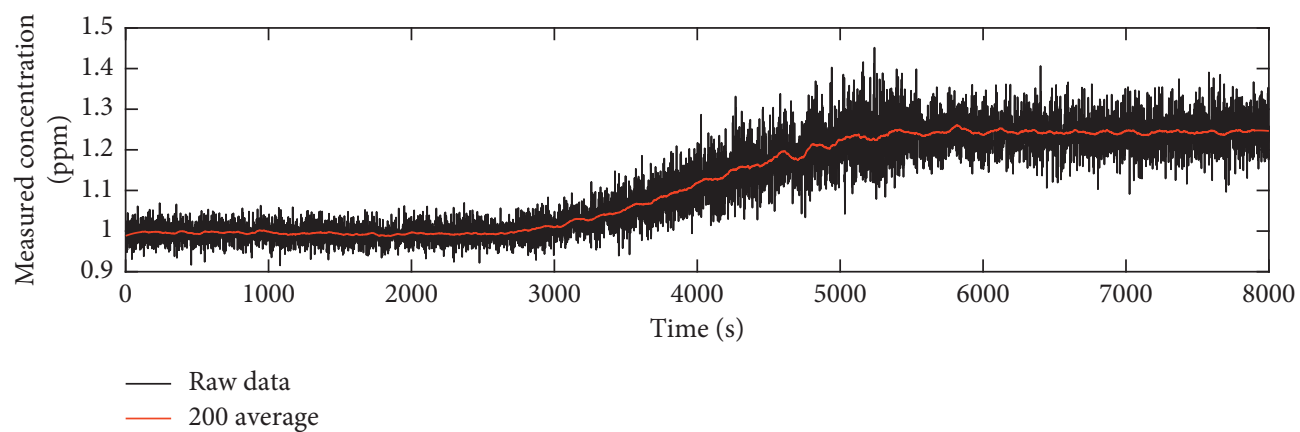

(a)

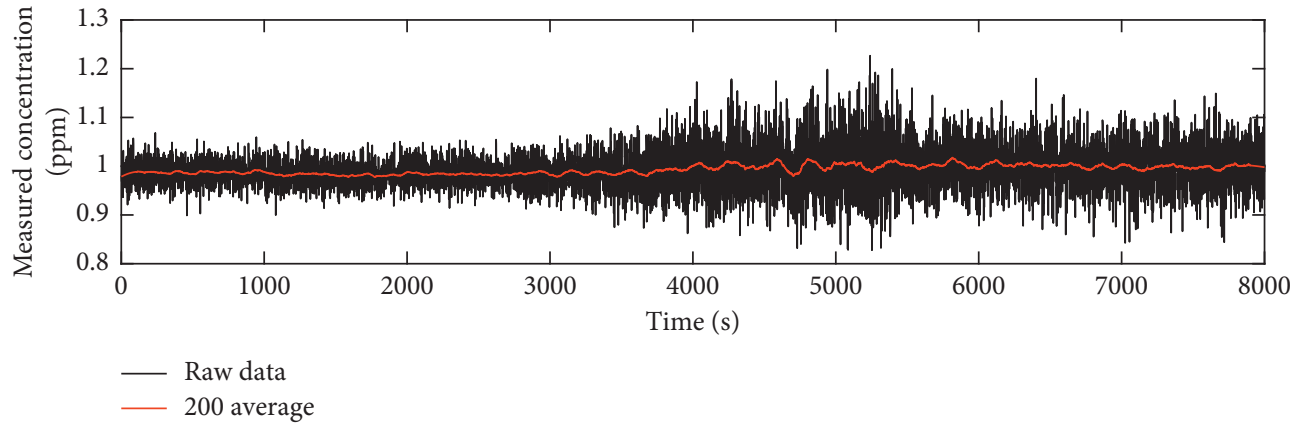

(b)

FIGURE 11: Comparison of detection results of $1 \mathrm{ppm}$ methane standard gas using the traditional method and differential measurement method.

\section{Discussion}

DAVAR is an extension of the classical Allan variance, which can not only meticulously characterize and identify the statistical characteristics of various error sources in CRDS detection but also analyze the dynamic characteristics of signals over time. The DAVAR is helpful to analyze the time-varying characteristics of nonstationary signals in the application of cavity ring-down spectroscopy in vehicle, airborne, and other harsh environments, so as to more intuitively characterize the correlation between environmental changes and detection accuracy.

The time-varying error characteristics reflected by DAVAR can be corrected by applying a differential measurement method. In this paper, a differential measurement method is used to correct the influence of temperature change on the detection accuracy. The differential measurement method herein improves the detection accuracy by detecting the "GROUND" of the CRDS device in real time and performing online calibration. 
TABle 1: Parameters used in the Voigt function approximation method.

\begin{tabular}{ccccc}
\hline & $A$ & $B$ & $C$ & $D$ \\
\hline 1 & -1.2150 & 1.2359 & -0.3085 & 0.0210 \\
2 & -1.3509 & 0.3786 & 0.5906 & -1.1858 \\
3 & -1.2150 & -1.2359 & -0.3085 & -0.0210 \\
4 & -1.3509 & -0.3786 & 0.5906 & 1.1858 \\
\hline
\end{tabular}

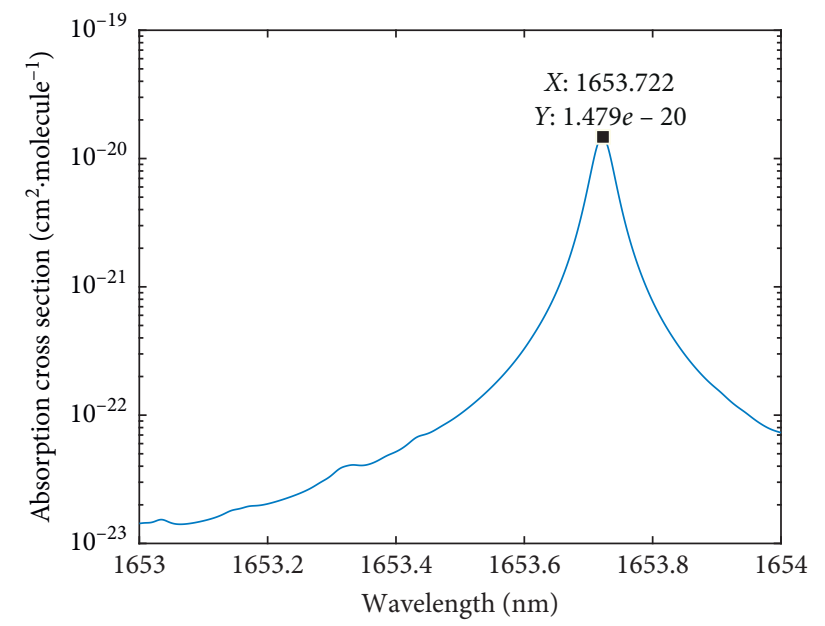

(a)

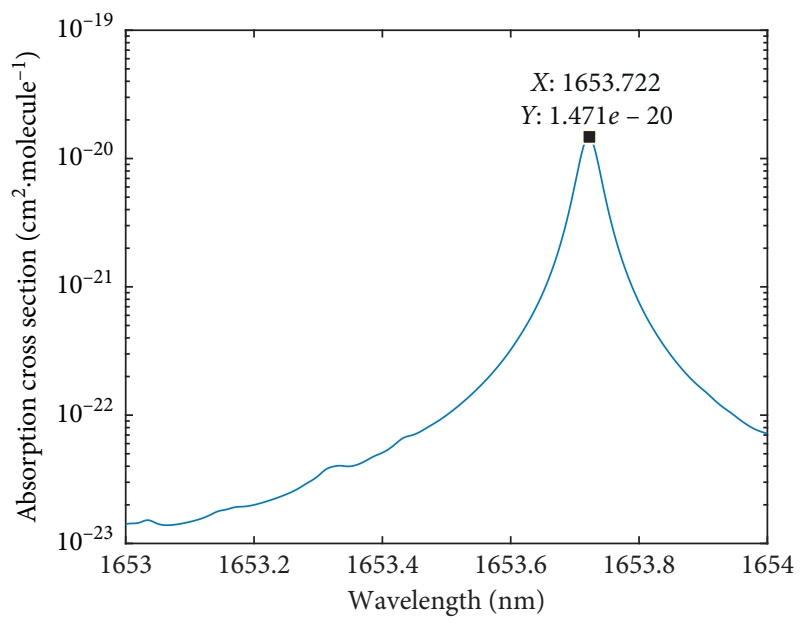

(b)

Figure 12: Effect of temperature change on methane absorption cross section at (a) $25^{\circ} \mathrm{C}$ and (b) $28^{\circ} \mathrm{C}$.

A stable "GROUND" is easy to obtain in a laboratory environment, but it is difficult to guarantee a stable detection environment in some practical CRDS technical solutions and applications. For example, it is a good detection solution for aerosols and corrosive gases using an open cavity, but the open cavity structure makes the detection device susceptible to the ambient. Moreover, in CRDS detection devices based on ball-borne platforms and UAV platforms, strict control of pressure and temperature requires additional power consumption and precise mechanical design, which will limit the application of CRDS. As mentioned above, in the CRDS equipment of Crosson [3], the temperature control accuracy is required to a few hundredths of a $K$. It can be seen that, from the experiments described herein, the practical differential measurement method can reduce the requirement on temperature control accuracy. The improved differential measurement method can reduce the requirements on the operating environment and thus improve the environmental adaptability of the instrument.

This method is not applicable in all cases. The differential measurement method requires detect the ring-down time at the absorption peak and the nonabsorption peak, respectively, which reduces the detection rate. Secondly, for the gas to be measured with a wide absorption peak, the current of DFB laser needs to be adjusted in a wide range to make the wavelength deviate from the absorption peak. This process will affect the thermal stability of the laser and may lead to the deterioration of the stability of the wavelength. Due to the above characteristics, the application of differential correction method in fast detection and the detection accuracy of gas with wide absorption cross section are limited.

\section{Conclusions}

In this paper, the DAVAR method is used to simulate the time-domain error in CRDS, and a differential detection method is proposed to correct the baseline drift in real time. With the numerical simulation of the influence of instabilities of sudden, slow, or periodic changes on the measurement accuracy in the ring-down process, DAVAR is proved to be an effective way to evaluate random error characteristics in an interfering environment. In this paper, a practical differential measurement method is proposed to correct the time-varying error by detecting ring-down times at the absorption peak and nonabsorption peak. A CRDS experimental device was constructed, and DAVAR was used to analyze the influence of cavity temperature change on detection accuracy. The effect of temperature change is corrected in real time by differential measurement, and the detection accuracy is improved about 19 times. The differential measurement method can be used to correct the time-varying error in real time and is helpful to improve the environmental adaptability of the CRDS instrument.

\section{Future Work}

Dynamic Allan variance analysis method and differential measurement method based on cavity decay spectrum will 
be used in open-path CRDS. The open cavity has great advantages in rapid detection of ambient gas and detection of extinction coefficient of aerosol [31,32]. However, due to the open structure, the cavity is more susceptible to the interference of the external environment. DAVAR can be used to analyze the impact of environmental changes on the detection accuracy, and the differential measurement method improves the environmental adaptability of CRDS instruments.

\section{Appendix}

The premise of the differential measurement calculation using equation (7) in the experiment of this paper is that the effect of temperature change on the gas absorption cross section is negligible. The Voigt linear combination with the HIRAN database was used to calculate the methane absorption cross sections at different temperatures [30]. The specific calculation process is as follows.

Voigt line is the convolution of Gaussian line and Lorentz line and can be expressed as follows:

$$
\begin{aligned}
& f_{v}\left(\nu-v_{0}\right)=A \frac{2 \ln 2}{\pi^{3 / 2}} \frac{\gamma_{L}}{\gamma_{G}^{2}} \\
& \int_{-\infty}^{+\infty} \frac{-e^{-t^{2}}}{\left(\sqrt{\ln 2}\left(\gamma_{L} / \gamma_{G}\right)\right)^{2}+\left(\sqrt{4 \ln 2}\left(\left(v-v_{0}\right) / \gamma_{G}\right)-t\right)^{2}} \mathrm{~d} t,
\end{aligned}
$$

where $\gamma_{L}$ is the FWHM (the full width half maximum) of the Lorentz line, $\gamma_{G}$ is the FWHM of the Gaussian line, and $A$ is the amplitude parameter. However, the function is not inferable, and it is processed here using the discrete method.

$$
\begin{aligned}
& f_{V}\left(\nu-v_{0}\right)=\frac{2}{\gamma_{G}} \sqrt{\frac{\ln 2}{\pi}} V(X, Y), \\
& \gamma_{G}=2 \frac{v}{c} \sqrt{\frac{2 \ln 2 N_{A} k T}{M}}, \\
& \gamma_{L}=2\left(\frac{T_{0}}{T}\right)^{n_{\text {air }}}\left[\gamma_{\text {air }} \cdot\left(p-p_{s}\right)+\gamma_{\text {self }} \cdot p_{s}\right],
\end{aligned}
$$

where $M$ is the relative molecular mass of the gas; $n_{\text {air }}$ is the temperature dependence coefficient; $\gamma_{\text {air }}$ is the air-broadened width; $\gamma_{\text {self }}$ is the self-broadened width; $p$ is the pressure, and $p_{s}$ is the partial pressure of the gas. The pressure in the HITRAN database is a standard atmospheric pressure, so the actual pressure is calculated as a ratio to the standard atmospheric pressure.

$$
\begin{aligned}
V(X, Y) & =\frac{Y}{\pi} \int_{-\infty}^{+\infty} \frac{e^{-z^{2}}}{Y^{2}+(X-z)^{2}} \mathrm{~d} z, \\
X & =\frac{2 \sqrt{\ln 2}}{\gamma_{G}}\left(\nu-\nu_{0}\right), \\
Y & =\frac{\gamma_{L}}{\gamma_{G}} \sqrt{\ln 2} .
\end{aligned}
$$

Since $V(X, Y)$ has no analytical solution, approximate calculations are used. The maximum deviation of the approximate calculation results is less than $5 \times 10^{-4}$.

$$
V(X, Y)=\sum_{i=1}^{4} \frac{C_{i}\left(Y-A_{i}\right)+D_{i}\left(X-B_{i}\right)}{\left(Y-A_{i}\right)^{2}+\left(X-B_{i}\right)^{2}} .
$$

The parameters $A_{\mathrm{i}}, B_{i}, C_{i}$, and $D_{i}$ are shown in Table 1 . Through MATLAB simulation, the absorption cross section of $R(3)$ at $1653.722 \mu \mathrm{m}$ with temperature was obtained, as shown in Figure 12. Figure 12(a) is a methane absorption cross section at $25^{\circ} \mathrm{C}$; the absorption cross section is $1.479 \times 10^{-20} \mathrm{~cm}^{2} \cdot$ molecule ${ }^{-1}$ at the absorption peak of $1653.722 \mathrm{~nm}$, and Figure 12(b) is a methane absorption cross section at the temperature of $28^{\circ} \mathrm{C}$, and the absorption cross section is $1.471 \times 10^{-20} \mathrm{~cm}^{2} \cdot$ molecule $^{-1}$ at the absorption peak of $1653.722 \mathrm{~nm}$.

\section{Data Availability}

The data used to support the findings of this study are included within the article.

\section{Conflicts of Interest}

The authors declare no conflicts of interest.

\section{Authors' Contributions}

Z.M. and J.Y. conceptualized the study; Z.M. and J.H. were responsible for methodology; Z.M., J.Y., J.W., and J.H. prepared the original draft; Z.M., J.W., and S.D. were responsible for formal analysis; Z.M. and J.W. investigated the study, J.Y. acquired funding; Z.M. and Y,L. reviewed and edited the study.

\section{Acknowledgments}

This research was funded by "Strategic Priority Research Program" of the Chinese Academy of Sciences (grant number XDA17040511); Instrument Developing Project of the Chinese Academy of Sciences (grant number YJKYYQ20170035); and the Innovation Program of Academy of Opto-Electronics, Chinese Academy of Science (grant number Y70B15A13Y).

\section{References}

[1] B. Paldus and A. Kachanov, "Spectroscopic techniques: cavity-enhanced methods," in Springer Handbook of Atomic, Molecular, and Optical Physics, G. Drake, Ed., pp. 633-640, Springer, New York, NY, USA, 2006.

[2] A. O'Keefe and D. A. G. Deacon, "Cavity ring-down optical spectrometer for absorption measurements using pulsed laser sources," Review of Scientific Instruments, vol. 59, no. 12, pp. 2544-2551, 1988.

[3] E. R. Crosson, "A cavity ring-down analyzer for measuring atmospheric levels of methane, carbon dioxide, and water vapor," Applied Physics B, vol. 92, no. 3, pp. 403-408, 2008.

[4] Y. Chen, P. Mahaffy, V. Holmes et al., "Near infrared cavity ring-down spectroscopy for isotopic analyses of $\mathrm{CH}_{4}$ on 
future Martian surface missions," Planetary \& Space Science, vol. 105, pp. 117-122, 2014.

[5] W. Chen, K. Roslund, C. L. Fogarty et al., "Detection of hydrogen cyanide from oral anaerobes by cavity ring down spectroscopy," Scientific Reports-UK, vol. 6, p. 22577, 2016.

[6] A. J. Orrewing, F. D. Pope, M. I. Mazurenka, W. H. Howie, I. C. Lane, and S. M. Newman, Applications of Cavity RingDown Spectroscopy in Atmospheric Chemistry, pp. 181-212, John Wiley \& Sons, Ltd, Chichester, UK, 2009.

[7] D. Liu, Z. Yu, and J. Lin, "Application of combustion module coupled with cavity ring-down spectroscopy for simultaneous measurement of SOC and $\delta^{13}$ C-SOC," Journal of Spectroscopy, vol. 2018, pp. 6893454-5, 2018.

[8] L. van der Sneppen, C. Gooijer, W. Ubachs, and F. Ariese, Cavity Ring-Down Spectroscopy in Analytical Chemistry, John Wiley \& Sons, Ltd, Chichester, UK, 2009.

[9] H. Huang and K. Lehmann, "Noise in cavity ring-down spectroscopy caused by transverse mode coupling," Optics Express, vol. 15, no. 14, p. 8745, 2007.

[10] D. Wang, R. Hu, P. Xie, M. Qin, L. Y Ling, and J. Dua, "Fast and accurate extraction of ring-down time in cavity ringdown spectroscopy," Spectroscopy and Spectral Analysis, vol. 34, pp. 2845-2850, 2014.

[11] Z.-q. Tan, Z.-g. Wang, and X.-w. Long, "A new theoretical derivation of continuous-wave cavity ring-down technology and its application emulation," Acta Photonica Sinica, vol. 36, pp. 60-63, 2007.

[12] C. Stowasser, A. D. Farinas, J. Ware et al., "A low-volume cavity ring-down spectrometer for sample-limited applications," Applied Physics B, vol. 116, no. 2, pp. 255-270, 2014.

[13] H. Huang and K. K. Lehmann, "Long-term stability in continuous wave cavity ringdown spectroscopy experiments," Applied Optics, vol. 49, no. 8, p. 1378, 2010.

[14] D. W. Allan, "Statistics of atomic frequency standards," Proceedings of the IEEE, vol. 54, no. 2, pp. 221-230, 1966.

[15] L. C. Ng and D. J. Pines, "Characterization of ring laser gyro performance using the allan variance method," Journal of Guidance, Control, and Dynamics, vol. 20, no. 1, pp. 211-214, 1997.

[16] L. Galleani and P. Tavella, "The characterization of clock behavior with the dynamic Allan variance," in Proceedings of the IEEE International Frequency Control Sympposium and PDA Exhibition Jointly with the 17th European Frequency and Time Forum, pp. 239-244, IEEE, Tampa, FL, USA, March 2003.

[17] L. Galleani and P. Tavella, "Tracking nonstationarities in clock noises using the dynamic Allan variance," in Proceedings of the 2005 IEEE International Frequency Control Symposium and Exposition, pp. 392-396, IEEE, Vancouver, Canada, January 2005.

[18] L. Galleani and P. Taveila, "Interpretation of the dynamic Allan variance of nonstationary clock data," in Proceedings of the IEEE International Frequency Control Symposium Joint with the 21st European Frequency and Time Forum, pp. 992-997, IEEE, Geneva, Switzerland, May 2007.

[19] J. Karhu, K. Lehmann, M. Vainio, M. Metsälä, and L. Halonen, "Step-modulated decay cavity ring-down detection for double resonance spectroscopy," Optics Express, vol. 26, no. 22, p. 29086, 2018.

[20] R. Engeln, G. Berden, R. Peeters, and G. Meijer, "Cavity enhanced absorption and cavity enhanced magnetic rotation spectroscopy," Review of Scientific Instruments, vol. 69, no. 11, pp. 3763-3769, 1998.
[21] M. Mazurenka, R. Wada, A. J. L. Shillings, T. J. A. Butler, J. M. Beames, and A. J. Orr-Ewing, "Fast Fourier transform analysis in cavity ring-down spectroscopy: application to an optical detector for atmospheric $\mathrm{NO}_{2}$," Applied Physics $B$, vol. 81, no. 1, pp. 135-141, 2005.

[22] L. Galleani and P. Tavella, “The dynamic Allan variance," IEEE Transactions on Ultrasonics, Ferroelectrics and Frequency Control, vol. 56, no. 3, pp. 450-464, 2009.

[23] I. Sesia, L. Galleani, and P. Tavella, "Application of the dynamic allan variance for the characterization of space clock behavior," IEEE Transactions on Aerospace and Electronic Systems, vol. 47, no. 2, pp. 884-895, 2011.

[24] Z. Na and L. Xuyou, "Research on theoretical improvement of dynamic Allan variance and its application," Acta Optica Sinica, vol. 31, no. 11, pp. 1106001-1106003, 2011.

[25] J. Courtois, K. Bielska, and J. T. Hodges, "Differential cavity ring-down spectroscopy," Journal of the Optical Society of America B, vol. 30, no. 6, p. 1486, 2013.

[26] J. Ye and J. L. Hall, "Cavity ringdown heterodyne spectroscopy: high sensitivity with microwatt light power," Physical Review A, vol. 61, no. 6, 2000.

[27] J. Benesty, J. Chen, Y. Huang, and I. Cohen, "Pearson correlation coefficient," in Noise Reduction in Speech Processing, Springer, Berlin, Germany, 2009.

[28] K. K. Lehmann, G. Berden, and R. Engeln, An Introduction to Cavity Ring-Down Spectroscopy, John Wiley \& Sons, Ltd, Chichester, UK, 2009.

[29] I. E. Gordon, L. S. Rothman, C. Hill et al., "The HITRAN 2016 molecular spectroscopic database," Journal of Quantitative Spectroscopy and Radiative Transfer, vol. 203, pp. 3-69, 2017.

[30] L. Xiuying, "Comparison analysis of atmospheric methane absorption cross section," in Proceedings of the 2012 International Conference on Computer Application and System Modeling, July 2012.

[31] L. E. Mchale, B. Martinez, T. W. Miller, and A. P. Yalin, "Open-path cavity ring-down methane sensor for mobile monitoring of natural gas emissions," Optics Express, vol. 27, no. 14, p. 20084, 2019.

[32] T. D. Gordon, N. L. Wagner, M. S. Richardson et al., "Design of a novel open-path aerosol extinction cavity ringdown spectrometer," Aerosol Science and Technology, vol. 49, no. 9, pp. 717-726, 2015. 